\title{
Considerations about Non-Metastatic Bladder Cancer Management During the COVID-19 Pandemic
}

\author{
Filipe L.F. Carvalho ${ }^{\mathrm{a}, \mathrm{b}, *}$, Lan Anh S. Gallowayc ${ }^{\mathrm{c}}$, Ragheed Saoud ${ }^{\mathrm{d}}$, Piyush K. Agarwal ${ }^{\mathrm{d}}$ \\ and Lambros Stamatakis ${ }^{\mathrm{a}, \mathrm{b}}$ \\ ${ }^{\mathrm{a}}$ MedStar Georgetown University Hospital, Washington, DC, USA \\ ${ }^{\mathrm{b}}$ MedStar Washington Hospital Center, Washington, DC, USA \\ ${ }^{\mathrm{c}}$ Georgetown University School of Medicine, Washington, DC, USA \\ ${ }^{\mathrm{d}}$ Department of Surgery, The University of Chicago, Section of Urology, Chicago, IL, USA
}

Received 9 April 2020

Accepted 5 May 2020

Pre-press 15 May 2020

Published 11 June 2020

\begin{abstract}
Bladder cancer is the sixth most common malignancy in the United States and is associated with high morbidity and mortality rates. To date, no guidelines have been published that clearly establish bladder cancer management recommendations during the COVID-19 pandemic. In this short commentary, we strive to outline recommendations for treatment of both muscle invasive and non-muscle invasive disease based on data from various trials and prior studies while taking into account the potential lack of hospital resources available to physicians depending on their practice location.
\end{abstract}

\section{INTRODUCTION}

COVID-19 is rapidly changing the landscape of medical care in 2020. Hospital resources are stretched thin as the number of cases continue to climb daily. However, with so many resources being diverted for this national pandemic, it begs the question: how can we continue to treat patients with other life-threatening illnesses during this time without compromising the necessary resources to manage a pandemic and putting our patients, as well as their families and local communities, at risk of contracting this virus?

Bladder cancer is one of the most common genito-urinary malignancies and is associated with

\footnotetext{
${ }^{*}$ Correspondence to: Filipe L.F. Carvalho, MedStar Georgetown University Hospital, Washington, DC, USA. E-mail: filipe.decarvalho@gunet.georgetown.edu.
}

significant morbidity and mortality, particularly when treatment is delayed. In addition, regardless of the tumor stage, bladder cancer treatment and follow up require a magnitude of resources that may be difficult to allocate during the COVID-19 pandemic. In response to this global pandemic, the American College of Surgeons recommended cancelation of non-urgent elective surgeries and the American Society for Clinical Oncology recommended postponing visits for patients not on active cancer treatment. Recent recommendations were subsequently made regarding the triage of urologic surgeries and in general, non-surgical treatments or deferral of surgery should be highly considered if possible [1]. This guidance is particularly challenging in non-muscle invasive bladder cancer (NMIBC) patients that need frequent cystoscopies, transurethral resections, and office visits. Moreover, delaying timely definitive therapy for muscle-invasive bladder cancer (MIBC) 
may result in missing the window of opportunity for cancer cure. Our goal is to discuss and suggest management of non-metastatic bladder cancer patients during this outbreak.

\section{NON-MUSCLE INVASIVE BLADDER CANCER}

Almost $80 \%$ of patients with newly diagnosed bladder cancer present with a non-muscle invasive tumor. NMIBC is a heterogeneous disease with different rates of recurrence and progression. The American Urological Association/Society of Urologic Oncology developed a risk stratification system that classifies NMIBC based on tumor grade, size, variant histology, lymphovascular invasion, multifocality, and response to intravesical BCG and tumor recurrence [2]. This system categorizes NMIBC into low, intermediate and high risk for recurrence and progression.

\section{Surveillance and TURBT}

Several studies have shown that low risk NMIBC can safely be managed with active surveillance following initial TURBT. Although the risk of recurrence may be as high as $61 \%$ at one year and $78 \%$ at 5 years, the risk of progression to muscle invasive bladder cancer is less than $1 \%$ at both one and five years [3]. A recent publication from the International Bladder Cancer Group (IBCG) provided recommendations for management of lowgrade NMIBC (including active surveillance) with the goals of reducing patient morbidity and improving system-wide resource utilization [4]. Given the inevitable shift of resources diverted to treat patients with COVID-19 and to prevent viral exposures during medical visits, we propose surveillance for all patients with a history of low-risk tumors - defined as low grade solitary Ta tumor $\leq 3 \mathrm{~cm}$ or papillary urothelial neoplasm of low malignant potential (PUNLMP) - and only performing TURBTs for symptomatic patients (e.g. intractable gross hematuria) during the pandemic, which is consistent with the aforementioned IBCG recommendations. If a TURBT is performed for suspected low-risk disease, an immediate perioperative dose of intravesical chemotherapy should be considered given the wellestablished reduction in tumor recurrence rates with this therapy $[5,6]$. We personally favor intravesical gemcitabine given the relative low cost, minimal safety concerns, and acceptable patient tolerability.
The intermediate risk group is more heterogeneous, hence more challenging to address during the current pandemic. Although the recurrence rate of intermediate risk tumors at 1 year is $47 \%$ and 5year recurrence free survival (RFS) is $33 \%$, rates of tumor progression to MIBC are approximately $2 \%$ at one year and more than $20 \%$ at 5 years $[7,8]$. Of note, solitary high grade (HG) Ta tumors $\leq 3 \mathrm{~cm}$ are categorized as intermediate risk by AUA guidelines, but other governing bodies like EAU and IBCG classify any tumor with HG features as "high risk." $[2,9,10]$. These HG Ta $\leq 3 \mathrm{~cm}$ tumors have RFS and PFS rates in between those quoted for the AUA intermediate and high-risk groups, thus confirming that the high-grade component is associated with a more aggressive tumor phenotype [8]. Nevertheless, given the relatively low risk of progression for low grade intermediate risk tumors, we also recommend surveillance for this group during the COVID-19 epidemic with consideration for TURBT for symptomatic patients only. In contrast, patients with $\mathrm{HG}$ Ta $\leq 3 \mathrm{~cm}$ tumors should be treated the same as high risk tumors (see below). Urologists may have to modify their treatment strategy if the associated restrictions on elective surgery persist beyond a reasonable time period with consideration for TURBT if symptoms develop or tumor progression is identified.

Large high grade Ta lesions and T1 tumors carry a significant risk of progression to MIBC. Up to $40 \%$ of HG Ta tumors progress to T1 and $25 \%$ to MIBC within 5 years [11]. Most T1 tumors are high grade with significant potential for stage progression if left untreated with up to $48 \%$ of patients progressing to $\mathrm{T} 2$ or greater $[12,13]$. For these reasons, we believe that patients with a diagnosis of high risk NMIBC should proceed with standard management at this time.

Many institutions are developing surgical review committees to triage which surgical cases should proceed based on recommendations from the American College of Surgeons. Given that a TURBT has a low risk of requiring admission, ICU resources, or aerosolization (i.e. compared to a patient undergoing ENT surgery), we recommend prioritizing these cases when presenting them to surgical review committees. Due to potential shortage of ventilators and to avoid exposure to aerosolized virus during intubation, the urology and anesthesia teams can consider performing selected cases under spinal anesthesia and/or sedation. Of note, there are likely regional differences in anesthesia preferences, although in our experience at our institution, the anesthesiologists prefer to intubate these patients as secretions and aerosoliza- 
tion are difficult to control in patients with regional anesthesia or general anesthesia without airway control.

The AUA guidelines strongly recommend repeat TURBT in patients with initial pathologic staging of high grade T1 disease, as up to $30 \%$ of such patients can be found to have MIBC on repeat resection. Moreover, repeat TURBT results in improvement in oncologic outcomes despite initial accurate primary tumor staging [14]. Some governing bodies have also advocated for consideration of repeat TURBT in patients with an initial diagnosis of high-grade $\mathrm{Ta}$ disease.

In the current COVID-19 era, we continue to advocate for repeat TURBT in patients with an initial diagnosis of high grade $\mathrm{T} 1$ disease, particularly if detrusor muscle could not be identified in the initial TURBT specimen. However, surgeon discretion, the nuances of a particular patient's case (i.e. relatively small initial tumor with a visually complete TURBT), and an institution's competing burden of COVID-19 cases all may contribute to a decision to delay and/or forgo a repeat TURBT. In such a scenario, extra attention should be paid to the patient's surveillance and response to intravesical therapy (see next section). In addition, repeat TURBT can likely be delayed or avoided for most patients with an initial diagnosis of high-grade Ta disease and an otherwise visually complete resection.

\section{Intravesical therapy}

Another equally important aspect of the management of high risk NMIBC is administration of intravesical therapies. Intravesical BCG therapy is the standard-of-care for high-risk NMIBC patients. Several clinical trials and meta-analyses have shown that intravesical BCG reduces recurrence rates and prevents progression to MIBC more effectively than any other current intravesical therapy. Level I evidence shows that maintenance BCG therapy has also been demonstrated to decrease tumor recurrences compared to a single 6-week induction course [15].

In 2019 , the urologic community faced a global BCG shortage and several reports were published on BCG allocation during this shortage. In short, patients with initial high risk NMIBC were prioritized to receiving BCG induction, particularly those with high grade $\mathrm{T} 1$ disease. Alternative intravesical chemotherapy was recommended if BCG was not available while early radical cystectomy (RC) was advocated in the highest risk cases. The Bladder
Cancer Advocacy Network (BCAN) expert panel recently discussed these issues in the face of the COVID-19 epidemic and suggested that it would be safe to delay the 5th and 6th doses of a BCG induction course for several weeks in order to avoid the risks of viral exposure during these clinic visits [16]. When faced with a patient on maintenance BCG, the panel recommended administering doses one and two of a three-week maintenance and skipping dose three entirely. Of note, BCAN's recommendations are based on BCG and do not comment on other intravesical treatments such as gemcitabine/docetaxel.

While these notions were proposed by bladder cancer thought leaders, they certainly have not been tested in a scientific manner to determine clinical equipoise with our current standard of care intravesical regimens. Ideally, high risk patients should receive an induction course of BCG followed by 2 maintenance courses (assuming complete response to the induction course). Consideration could be given to administring an abbreviated course of intravesical therapy depending on the presumed cancer risk, BCG supply and the current status of COVID-19 epidemic within the local community. We feel this recommendation should apply to other courses of intravesical chemotherapy but realize that the data to support the efficacy of such an abbreviated course of therapy is lacking.

\section{MUSCLE-INVASIVE BLADDER CANCER}

MIBC is a more lethal disease that requires prompt treatment. If left untreated, its cancer-specific mortality (CSM) at 6, 9 and 12 months is $41 \%, 50 \%$ and $58 \%$, respectively [17]. Data by Gore et al. have shown that delays in treatment can be detrimental. When RC was delayed for 12 weeks after diagnosis the hazard ratio of death was twice as great as those who had their cystectomy done between 4 and 8 weeks after diagnosis [18]. If RC is delayed more than 12 weeks from diagnosis, the risk of death increases 7.7-fold at two-year follow-up.

While few would argue that timely RC for MIBC is crucial for patients pursuing surgery, the COVID19 pandemic has forced the urologic community to reconsider this paradigm and potentially alter traditional decision making. The estimated national average length of stay (LOS) after RC has been reported to be 11 days [19]. Institutions with higher RC volume and with established ERAS protocols have demonstrated lower LOS, with an average stay 
of 5 days. While most patients have an uncomplicated course, $16 \%$ of patients require at least 1 day in the ICU and almost $10 \%$ are discharged to a skilled nursing facility or rehabilitation center for further care after their acute hospitalization [19, 20]. The potential need for ICU care after RC must be taken into consideration during the COVID-19 pandemic.

Major surgeries like RC are resource-intensive with the need for ventilators, masks, and other equipment. Moreover, the need for human resources is extensive and something that may be taken for granted. Obviously, the OR staff is necessary for such a case to be performed, but the preoperative staff, the admissions staff, the postoperative staff, the sterilization processing staff, the ward staff, the janitorial staff, etc., also provide vital contributions to the workflow. General reductions in surgical volume translate into a reduction in interactions amongst personnel within a hospital system, which may help reduce viral transmission during this pandemic. These factors all need to be considered during this time when material and human resources may be scarce and necessitate diversion to other healthcare areas.

With these factors in mind, each individual patient's case needs to be scrutinized for necessity and scheduling prioritization. We believe that $\mathrm{RC}$ is one of the procedures that should be considered urgent enough to perform during the COVID-19 pandemic. Certainly, ERAS protocols should be implemented to allow earlier hospital discharge. Telemedicine should be used when feasible for pre- and postoperative visits. As per BCAN recommendations, incision and stoma care may be monitored by urologists through a video visit. In addition, externalized ureteral stents could be removed by the patient or a caregiver with video supervision. Frequent remote "check-ins" with the patient should be performed by providers or nurse navigators to encourage patient hydration and mobilization and provide early identification of problems that could potentially be handled in the urology office as opposed to the emergency department or other locations of potential high viral exposure. In fact, the urology community could consider adoption of telemedicine visits for routine monitoring even after the pandemic ends.

\section{Considerations for perioperative chemotherapy}

Multiple studies have shown that neoadjuvant chemotherapy (NAC) is associated with improvement in overall survival (OS), decreased lymph node metastasis, decreased recurrence rates and pathologic downstaging, without a significant increase in morbidity [21-23]. Although cisplatin-based regimens of NAC followed by RC have been the standard treatment for cT2-T4a node negative MIBC for almost ten years, NAC remains underused due to provider misperceptions about the benefits and fear of chemotherapeutic toxicity [24].

On the other hand, outcomes of patients receiving chemotherapy who become infected with COVID-19 remain uncertain and may increase their mortality risk. While there is a paucity of data regarding outcomes of cancer patients infected with COVID19 , recent reports suggest that patients on active cancer treatment have worse complications. Data from a small cohort of 28 cancer patients treated at Wuhan, China (one of the early epicenters of the virus) revealed that patients receiving anti-tumor therapy, including chemotherapy, radiation therapy, targeted therapy and immunotherapy demonstrated poor outcomes after COVID-19 infection [25]. This retrospective study highlighted that $53.6 \%$ developed severe events, $21.4 \%$ required ICU admission, and $35.7 \%$ had life-threatening complications. Ultimately, $28.6 \%$ of this cohort died. Patients that were infected with COVID-19 within 14 days of receiving anti-tumor therapies were significantly more likely to have severe events from COVID-19 infection. These results need further validation from larger studies and will hopefully improve our understanding of the effects of this infection on different cancer types and scenarios, such as early vs advanced stage and types of therapies.

Our recommendation is to risk stratify patients with MIBC taking into account the pandemic status of the community where patients will be treated. Even though there is level one evidence supporting NAC in all patients with MIBC (cT2-T4), we propose upfront $\mathrm{RC}$ for surgically-fit patients who are deemed to have organ-confined disease, particularly those with a large tumor burden and local symptoms (persistent gross hematuria, LUTS, etc) [26]. This approach follows the recently published guidelines from the EAU, American College of Surgeons, and the Centers for Medicaid/Medicare Services, where surgery for "high risk" tumors in all specialties is considered high acuity ("Tier 3a") and should not be postponed if resources are available. Conversely, in patients with locally advanced primary tumors (cT3-4) or those presenting to healthcare systems overwhelmed with COVID-19 cases, NAC should be prioritized. 


\section{BLADDER-SPARING TRIMODALITY TREATMENT}

Bladder-sparing trimodality therapy (TMT) represents an attractive alternative to radical cystectomy in patients with MIBC who elect to retain their native bladder. Although there are no clinical trials directly comparing TMT and RC, studies have shown comparable OS and disease-specific survival (DSS) between these two treatment modalities [27]. Ideal candidates include those patients with a unifocal tumor confined to the bladder (cT2) who have all grossly visible tumor resected by TURBT, and do not exhibit associated hydronephrosis or CIS at sites topographically separate from the primary tumor bed. Patients who fit these criteria can expect a DSS approaching $84 \%$ at 10 years with an associated cystectomy rate of less than $15 \%$ over this time period [27]. Salvage cystectomy after failed TMT has acceptable surgical morbidity and outcomes [28]. Interestingly, variant histology does not appear to significantly influence outcomes after TMT and should not be a contraindication for pursuing TMT [29]. In addition, TMT is a very appealing option for elderly patients that might not be fit for or interested in undergoing major surgery. Moreover, older individuals seem to exhibit a higher risk of severe complications and associated mortality with COVID-19 infection. As such, TMT should be considered an appropriate alternative to RC in select patients with MIBC, particularly in older patients and/or those with medical comorbidities that predict a higher risk of postoperative complications or a prolonged convalescence.

\section{SYSTEMIC IMMUNOTHERAPY}

In recent years, five different immunomodulators targeting program cell death 1 (PD-1) - pembrolizumab and durvalumab - and programmed cell death-ligand 1 (PD-L1) - atezolizumab, nivolumab, and avelumab - pathways have been FDA approved for the treatment of bladder cancer. All these drugs were initially approved as second line therapies for patients with metastatic bladder cancer who have progressed after cisplatin-based chemotherapy, but the clinical efficacy and safety profile of these agents made them appealing for use in multiple other disease states, including NMIBC. A singlearm open-label phase 2 study — KEYNOTE-057 enrolled patients with BCG-unresponsive high risk NMIBC (specifically those with carcinoma in situ with or without associated papillary tumor) to receive pembrolizumab every 3 weeks. Results from this study demonstrated a complete response $(\mathrm{CR})$ rate of approximately $40 \%$ at 3 months [30]. Of those patients who achieved a CR, approximately 59\% maintained a CR at a median follow-up of 16.7 months (range 5.9-28.2 months). At the time of publication, none of the patients in the study progressed to muscle invasive or metastatic disease. Moreover, the treatment was very tolerable with an acceptable safety profile with only $12.7 \%$ of subjects suffered a Clavien-Dindo grade 3 or 4 complication. These results led to the FDA approval of pembrolizumab to treat patients BCG-unresponsive high risk NMIBC with CIS [31].

The AUA guidelines recommend strong consideration for radical cystectomy in patients with BCG-unresponsive high risk NMIBC. In light of this recent drug approval, we believe it is reasonable to propose treatment with pembrolizumab as an alternative to radical cystectomy during the pandemic. Salvage intravesical chemotherapy (i.e. intravesical gemcitabine + docetaxel) can also be considered in this setting.

\section{CLINICAL TRIALS}

Currently, there are multiple clinical trials available for patients with NMIBC and MIBC that may be affected by COVID-19. To date there have been no federal guidelines published encouraging trial sponsors to delay or halt clinical trials. The FDA has released a statement encouraging sponsors to "consider each circumstance, focusing on the potential impact on the safety of trial participants, and modify study conduct accordingly" [32]. In addition, the FDA instructs trial sponsors to consider patient safety and allow protocol changes without IRB approval to "minimize or eliminate immediate hazards or to protect the life and well-being of research participants (e.g. to limit exposure to COVID-19)." The National Cancer Institute and the American Society of Clinical Oncology acknowledge that conducting clinical trials will be particularly challenging during this time and recommendations remain vague. Our recommendation is to have multidisciplinary discussions with patients and their caregivers and balance the potential benefits of the trial and risks of exposure to COVID-19. For patients that are already enrolled in therapeutic trials, all efforts should be made to continue providing the appropriate 


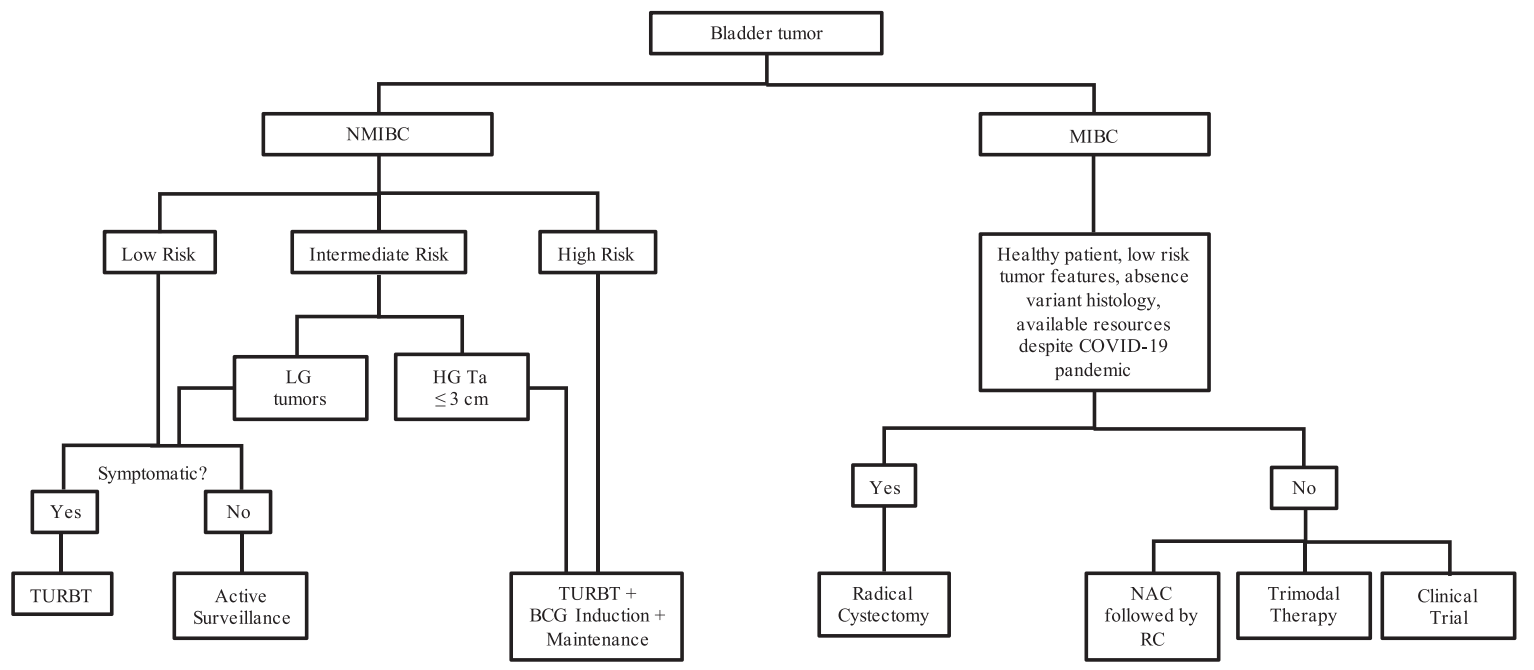

Fig. 1. Treatment algorithm for patients with bladder cancer during the COVID-19 pandemic. LG - low grade; HG - high grade; TURBT transurethral resection of bladder tumor; RC - radical cystectomy; NAC - neoadjuvant chemotherapy.

treatment. Providers and companies should consider, for example, shipping medication and assessment forms to patients' homes to avoid office visits. We should consider placing non-therapeutic trials focused on biomarker discovery or relying on tissue banking on hold. However, this period might provide an opportunity to complete non-interventional studies that do not require office visits such as QOL questionnaires and phone interviews. Enrollment of new patients or opening new therapeutic clinical trials should be made on a case-by-case basis with the guidance of local IRBs and clinical leadership.

\section{CONCLUSION}

As the COVID-19 pandemic continues to surge, we must consider how to care for and treat patients with both NMIBC and MIBC without decreasing already limited hospital resources. For NMIBC, we advocate for active surveillance for patients harboring low risk and most intermediate risk disease. We also suggest the continuation of standard-of-care therapy for high risk disease, including repeat TURBT for high grade $\mathrm{T} 1$ disease and use of intravesical BCG with potential course modifications.

For patients with MIBC, delays in treatment have been shown to be detrimental to outcomes. While each patient scenario needs to be individually scrutinized (i.e. symptoms, extent of disease, pros/cons of a specific therapy, likelihood of resource utilization and/or complications, current COVID-19 state within an institution, etc.), we generally recommend upfront
RC in surgically-fit patients with clinically localized MIBC, particularly if symptomatic. NAC should be encouraged for those with more locally advanced disease (cT3-T4). Bladder-sparing TMT is a perfectly acceptable alternative to $\mathrm{RC}$ in well-selected patients.

We encourage clinical trial investigators to consider novel approaches to patient monitoring and disease management. Looking forward, recommendations will inevitably need to evolve with the quickly changing landscape of medical care during the COVID-19 pandemic.

\section{ACKNOWLEDGMENTS}

The authors have no acknowledgments.

\section{FUNDING}

The authors report no funding.

\section{AUTHOR CONTRIBUTIONS}

FLFC: conception, performance of work, interpretation and analysis of data, writing the article; LASG: performance of work, interpretation and analysis of data, writing the article; RS: performance of work, interpretation and analysis of data, writing the article; PKA: conception, performance of work, interpretation and analysis of data, writing the article; LS: conception, performance of work, interpretation and analysis of data, writing the article. 


\section{ETHICAL CONSIDERATIONS}

Due to the nature of this manuscript - short review - our study is IRB exempt.

\section{CONFLICT OF INTEREST}

PKA: AstraZeneca Advisory Board (unpaid).

The other authors do not have any conflicts of interest to disclose.

\section{REFERENCES}

[1] Stensland KD, Morgan TM, Moinzadeh AT, Lee CT, Briganti A, Catto J, et al. Considerations in the triage of urologic surgeries during the COVID-19 Pandemic. Eur Urol. 2020Mar15; in press.

[2] Chang SS, Boorjian SA, Chou R, Clark PE, Daneshmand S, Konety BR, et al. Diagnosis and Treatment of Non-Muscle Invasive Bladder Cancer: AUA/SUO Guideline. 2016.

[3] Tiu A, Jenkins LC, Soloway MS. Active surveillance for low-risk bladder cancer. Urol Oncol: Seminars and Original Investigations. 2014;32(1):e7-e10.

[4] Matulay JT, Soloway M, Witjes JA, Buckley R, Persad R, Lamm DL, et al. Risk-adapted management of low-grade bladder tumours: recommendations from the International Bladder Cancer Group (IBCG). BJU Int. 2020Aug;125(4):497-505.

[5] Sylvester RJ, Oosterlinck W, Holmang S, Sydes MR, Birtle A, Gudjonsson S, et al. Systematic Review and Individual Patient Data Meta-analysis of Randomized Trials Comparing a Single Immediate Instillation of Chemotherapy After Transurethral Resection with Transurethral Resection Alone in Patients with Stage pTa-pT1 Urothelial Carcinoma of the Bladder: Which Patients Benefit from the Instillation? European Urology. 2016;69(2):231-44.

[6] Messing EM, Tangen CM, Lerner SP, Sahasrabudhe DM, Koppie TM, Wood DP, et al. Effect of Intravesical Instillation of Gemcitabine vs Saline Immediately Following Resection of Suspected Low-Grade Non-MuscleInvasive Bladder Cancer on Tumor Recurrence. JAMA. 2018Aug;319(18):1880.

[7] Ravvaz K, Weissert JA, Downs TM. American Urological Association Nonmuscle Invasive Bladder Cancer Risk Model Validation-Should Patient Age be Added to the Risk Model? J Urol. 2019;202(4):682-8.

[8] Ritch CR, Velasquez MC, Kwon D, Becerra MF, SoodanaPrakash N, Atluri VS, et al. Use and Validation of the AUA/SUO Risk Grouping for Nonmuscle Invasive Bladder Cancer in a Contemporary Cohort. J Urol. 2020;203(3):505-11.

[9] Babjuk M, Burger M, Compérat EM, Gontero P, Mostafid $\mathrm{AH}$, Palou J, et al. European Association of Urology Guidelines on Non-muscle-invasive Bladder Cancer (TaT1 and Carcinoma In Situ) - 2019 Update. European Urology. 2019;76(5):639-57.

[10] Kamat AM, Witjes JA, Brausi M, Soloway M, Lamm D, Persad R, et al. Defining and Treating the Spectrum of Intermediate Risk Nonmuscle Invasive Bladder Cancer. Journal of Urology. 2014;192(2):305-15.
[11] Herr HW. Tumor Progression and Survival of Patients with High Grade, Noninvasive Papillary (TaG3) Bladder Tumors: 15-Year Outcome. J Urol. 2000;163(1):60-2.

[12] Nieder AM, Brausi M, Lamm D, O'Donnell M, Tomita $\mathrm{K}$, Woo $\mathrm{H}$, et al. Management of stage $\mathrm{T} 1$ tumors of the bladder: International Consensus Panel. Urology. 2005;66(6):108-25.

[13] Pasin E, Josephson DY, Mitra AP, Cote RJ, Stein JP. Superficial Bladder Cancer: An Update on Etiology, Molecular Development, Classification, and Natural History. Rev Urol. 2008;10(1):31-43.

[14] Sfakianos JP, Kim PH, Hakimi AA, Herr HW. The Effect of Restaging Transurethral Resection on Recurrence and Progression Rates in Patients with Nonmuscle Invasive Bladder Cancer Treated with Intravesical Bacillus Calmette-Guérin. Journal of Urology. 2014;191(2):341-5.

[15] Lamm DL, Blumenstein BA, Crissman JD, Montie JE, Gottesman JE, Lowe BA, et al. Maintenance Bacillus Calmette-Guerin Immunotherapy For Recurrent Ta, T1 And Carcinoma In Situ Transitional Cell Carcinoma Of The Bladder: A Randomized Southwest Oncology Group Study. J Urol. 2000;163:1124-9.

[16] Banks R, Kamat AM, Gore JL, Lerner S, Shore $\mathrm{N}$, Singh $\mathrm{P}$, et al. COVID-19 and Bladder Cancer FAQ [Internet]. BCAN. Bladder Cancer Advocacy Network; 2020. Available from: https://bcan.org/covid-19faq/\#1585074665048-c86a0f20-0ab7

[17] Martini A, Sfakianos JP, Renström-Koskela L, Mortezavi A, Falagario UG, Egevad L, et al. The natural history of untreated muscle-invasive bladder cancer. BJU Int. 2019Jul;125(2):270-5.

[18] Gore JL, Lai J, Setodji CM, Litwin MS, Saigal CS. Mortality increases when radical cystectomy is delayed more than 12 weeks. Cancer. 2009Jan;115(5):988-96.

[19] Semerjian A, Milbar N, Kates M, Gorin MA, Patel HD, Chalfin HJ, et al. Hospital Charges and Length of Stay Following Radical Cystectomy in the Enhanced Recovery After Surgery Era. Urology. 2018;111:86-91.

[20] Pearl JA, Patil D, Filson CP, Arya S, Alemozaffar M, Master VA, et al. Patient Frailty and Discharge Disposition Following Radical Cystectomy. Clin Genitourin Cancer. 2017;15(4).

[21] Grossman HB, Natale RB, Tangen CM, Speights V, Vogelzang NJ, Trump DL, et al. Neoadjuvant Chemotherapy plus Cystectomy Compared with Cystectomy Alone for Locally Advanced Bladder Cancer. N Engl J Med. 2003;349(9):859-66.

[22] Vale C. Neoadjuvant Chemotherapy in Invasive Bladder Cancer: Update of a Systematic Review and Meta-Analysis of Individual Patient Data. Eur Urol. 2005;48:202-6.

[23] Kitamura H, Tsukamoto T, Shibata T, Masumori N, Fujimoto H, Hirao Y, et al. Randomised phase III study of neoadjuvant chemotherapy with methotrexate, doxorubicin, vinblastine and cisplatin followed by radical cystectomy compared with radical cystectomy alone for muscle-invasive bladder cancer: Japan Clinical Oncology Group Study JCOG0209. Ann Oncol. 2014;25(6): 1192-8.

[24] Reardon ZD, Patel SG, Zaid HB, Stimson C, Resnick MJ, Keegan KA, et al. Trends in the Use of Perioperative Chemotherapy for Localized and Locally Advanced Muscle-invasive Bladder Cancer: A Sign of Changing Tides. European Urology. 2015;67(1):165-70.

[25] Zhang L, Zhu F, Xie L, Wang C, Wang J, Chen R, et al. Clinical characteristics of COVID-19-infected cancer patients: 
A retrospective case study in three hospitals within Wuhan, China. Ann Oncol. 2020

[26] Ribal MJ, Cornford P, Briganti A, Knoll T, Gravas S, Babjuk $\mathrm{M}$, et al. European Association of Urology Guidelines Office Rapid Reaction Group: An Organisation-wide Collaborative Effort to Adapt the European Association of Urology Guidelines Recommendations to the Coronavirus Disease 2019 Era. European Urology. 2020; in press.

[27] Giacalone NJ, Shipley WU, Clayman RH, Niemierko A, Drumm M, Heney NM, et al. Long-term Outcomes After Bladder-preserving Tri-modality Therapy for Patients with Muscle-invasive Bladder Cancer: An Updated Analysis of the Massachusetts General Hospital Experience. European Urology. 2017;71(6):952-60.

[28] Eswara JR, Efstathiou JA, Heney NM, Paly J, Kaufman DS, Mcdougal WS, et al. Complications and Long-Term Results of Salvage Cystectomy After Failed Bladder Sparing Therapy for Muscle Invasive Bladder Cancer. Journal of Urology. 2012;187(2):463-8.

[29] Krasnow RE, Drumm M, Roberts HJ, Niemierko A, Wu $\mathrm{C}-\mathrm{L}, \mathrm{Wu} \mathrm{S}$, et al. Clinical Outcomes of Patients with Histologic Variants of Urothelial Cancer Treated with Trimodality Bladder-sparing Therapy. European Urology. 2017;72(1):54-60.
[30] Balar AV, Kulkarni GS, Uchio EM, Boormans JEM, Mourey LA, Krieger LF, et al. Keynote 057: Phase II trial of Pembrolizumab (pembro) for patients (pts) with high-risk (HR) nonmuscle invasive bladder cancer (NMIBC) unresponsive to bacillus calmette-guérin (BCG). Journal of Clinical Oncology. 2019Jan;37(7_suppl):350-350.

[31] FDA approves pembrolizumab for BCG-unresponsive, high-risk non-muscle invasive bladder cancer [Internet]. FDA approves pembrolizumab for BCG-unresponsive, high-risk non-muscle invasive bladder cancer FDA; Jan 8, 2020. Available from: https://www.fda.gov/drugs/ resources-information-approved-drugs/fda-approves-pemb rolizumab-bcg-unresponsive-high-risk-non-muscle-invasi ve-bladder-cancer

[32] FDA Guidance on Conduct of Clinical Trials of Medical Products during COVID-19 Pandemic: Guidance for Industry, Investigators, and Institutional Review Boards [Internet]. FDA Guidance on Conduct of Clinical Trials of Medical Products during COVID-19 Pandemic: Guidance for Industry, Investigators, and Institutional Review Boards Food and Drug Administration; Mar 18, 2020. Available from: https://www.fda.gov/media/136238/download 